Original Article Journal of Epilepsy Research pISSN 2233-6249 / elSSN 2233-6257

Received November 30, 2016 Accepted December 14, 2016

Corresponding author: Sang Kun Lee Department of Neurology, Seoul National University Hospital, 101 Daehak-ro, Jongno-gu, Seoul 03080, Korea

Tel. +82-2-760-2923

Fax. $+82-2-744-1785$

E-mail; sangkun2923@gmail.com

\section{Change of Patient Selection Strategy and Improved Surgical Outcome in MRI-negative Neocortical Epilepsy}

\author{
Hye-Jin Moon ${ }^{1}$, Dong Wook Kim², Chun-Kee Chung ${ }^{3}$, Jung-won Shin ${ }^{4}$, Jangsup Moon ${ }^{4}$, \\ Bong Su Kang ${ }^{5}$, Soon-Tae Lee ${ }^{4}$, Keun-Hwa Jung ${ }^{4}$, Kon Chu ${ }^{4}$, Ki-Young Jung ${ }^{4}$, \\ Yong Won $\mathrm{Cho}^{1}$, Sang Kun Lee ${ }^{4}$ \\ ${ }^{1}$ Department of Neurology, Keimyung University Dongsan Medical Center, Daegu; ${ }^{2}$ Department of Neurology, \\ Konkuk University School of Medicine; ${ }^{3}$ Department of Neurosurgery; ${ }^{4}$ Department of Neurology, Seoul National \\ University College of Medicine; ${ }^{5}$ Department of Neurology, Korea University College of Medicine, Seoul, Korea
}

Background and Purpose: It is crucial to make selection strategy to identify surgical candidates among medically refractory MRI-negative neocortical epilepsy patients. In our previous study, we suggested two or more concordance between noninvasive studies (EEG, ictal scalp EEG, interictal FDG-PET, and SPECT) as a new patient selection strategy for MRI-negative neocortical epilepsy surgery. The objective of this study was to evaluate the surgical outcomes of MRI-negative neocortical epilepsy patients before and after the implementation of a new selection strategy.

Methods: From 1995 to 2011, we included 153 consecutive MRI-negative neocortical epilepsy patients who received focal resection and had a follow-up period of at least 2 years. These patients were divided into two groups according to their date of surgery (before and after July 2002). The old group consisted of 89 patients and the new one consisted of 53 patients. Clinical characteristics, presurgical evaluations, and pathology were reviewed.

Results: The new patient selection strategy led to a significant increase in the concordance between two or more modalities. The improvement in surgical outcome after 2002 was significant (seizure-free outcome, $47.2 \%$ vs. $75.5 \% ; p=0.001$ ). Concordance between two or more presurgical evaluations and localizing PET were related to a seizure-free outcome in a multivariate analysis.

Conclusions: After a change in surgical strategy to select patients with two or more concordance between noninvasive studies, the seizure-free outcome improved up to $75.5 \%$. MRI-negative neocortical epilepsy patients with two or more concordance between noninvasive studies seem to be good candidates for epilepsy surgery. (2016;6:68-76)

Key words: MRI-negative neocortical epilepsy, Epilepsy surgery, Presurgical evaluation, Surgical outcome

\section{Introduction}

Resective surgery for medically refractory epilepsy is accepted as an effective management, especially for patients with a discrete lesion on magnetic resonance imaging (MRI). However, MRI is normal or nonspecific in a proportion of patients with partial epilepsy, even in patients with histopathologically confirmed lesions, such as cortical dysplasia. ' While a number of MRI-negative epilepsy patients turn out to be medically intractable, these patients have a lower chance of surgery than do those with discrete MRI lesions. ${ }^{2}$ Moreover, if they undergo epilepsy surgery, their surgical outcomes are generally poorer than those of MRI-positive patients. ${ }^{2-5}$ This reflects the difficulty in the accurate localization and complete resection of the epileptogenic zone in the absence of MRI-identified lesions. The definition of prognostic factors, including the results of multimodal diagnostic tools, is important to identify ideal candidates for surgery among MRI-negative patients.

Recently, the number of mesial temporal lobectomy for hippocampal sclerosis has reduced ${ }^{6}$ and the increase in neocortical epilepsy surgery was not significant. So this led to the overall reduction of epilepsy surgery, ${ }^{7}$ despite there is no evidence of reduction in the number of treatment-resistant epilepsy patients overall. ${ }^{8}$ The key fac- 
tor to hesitate the neocortical epilepsy surgery is the poor outcome of MRI-negative neocortical epilepsy. ${ }^{9}$ Identifying prognostic factors and selecting ideal surgical candidates for epilepsy surgery of MRI-negative patients is crucial.

In our previous study, ${ }^{10}$ we found that surgical treatment can benefit MRI-negative neocortical epilepsy patients, and that presurgical studies can localize correctly the epileptogenic lobe in these patients. In the same study, we showed that, among four presurgical evaluation modalities (ictal scalp electroencephalogram [EEG], interictal scalp EEG, fluorodeoxyglucose-positron emission tomography [FDG-PET], and ictal-interictal single-photon emission computed tomography [SPECT]), the concordance between two or more presurgical results was related to a seizure-free outcome. Subsequently, we have used this approach as a new strategy for the selection of MRI-negative neocortical epilepsy patients for surgery. The strategy consists in selecting refractory MRI-negative epilepsy patients who have two or more concordance among presurgical noninvasive modalities.

The objective of this study was to evaluate the surgical outcomes of MRI-negative epilepsy neocortical epilepsy patients before and after the implementation of the new patient selection strategy. We also defined prognostic factors, including the results of multimodal presurgical evaluation tools, and the diagnostic sensitivities of these tools using pooled data.

\section{Methods}

\section{Patients}

After obtaining approval from the Seoul National University Hospital (SNUH) review board, from a prospective database we retrospectively identified 153 consecutive patients without MRI-detectable lesions who underwent epileptic surgery at the SNUH between September 1995 and March 2011. As we applied a new patient selection strategy after July 2002, we classified these patients into two groups according to their surgery date. Before July 2002, we considered surgical treatment of MRI-negative neocortical epilepsy even when they had at least one localizing result of presurgical studies (ictal scalp EEG, interictal scalp EEG, FDG-PET, and ictal-interictal SPECT) with the consideration of seizure semiology, while our new patient selection strategy required concordant results of two or more presurgical studies. Although we tried to apply the new strategy, a few individuals who had lower concordance were included in this new group based on their invasive study results, because the final de- cision to take surgical treatment was usually made by the patient or their care-giver after the explanation of the possibility of successful surgery based on the results of presurgical studies. So we excluded 11 patients with low concordance (0-1) from the new group for comparison between old and new groups. The old group consisted of 89 patients and the new one consisted of 53 patients. Concordance of presurgical studies was defined as the results of ictal/interictal EEG and functional neuroimaging were localized to the same epileptogenic lobe. We included only patients who received focal resection and had a follow-up period of at least 2 years. The mean follow-up period was $11.4 \pm 5.16$ years for the old group and $4.2 \pm 1.65$ years for the new group. We excluded patients with epileptic surgery other than focal resection, such as functional hemispherectomy or corpus callosotomy. Patients with a medial temporal ictal onset zone were excluded.

\section{Presurgical evaluation}

A multidisciplinary presurgical evaluation was performed. All patients underwent a thorough history taking and physical/neurological examinations. All patients underwent brain MRI using an epilepsy protocol as described previously, ${ }^{10} \mathrm{MRI}$ was performed on 1.5-T or, since 2003, 3-T systems. Our imaging protocol included 3 $\mathrm{mm}$ T2-weighted/fluid-attenuated inversion recovery oblique coronal images and $1.5 \mathrm{~mm}$ T1-weighted three-dimensional magnetization-prepared rapid acquisition with gradient-echo sequences. An experienced neuroradiologist who was blinded to other presurgical evaluation findings viewed the MRI first. The secondary interpretations were performed at a multidisciplinary case conference based on data from video-EEG monitoring, FDG-PET, and ictal-interictal SPECT, if available.

Continuous video-EEG monitoring was performed in all patients, to capture at least three habitual seizures. The international 10-20 system and additional anterior temporal electrodes were used for scalp EEG monitoring. Interictal epileptiform discharges and ictal-onset rhythms were classified as localizing if they were confined to the electrodes of the epileptogenic lobe. According to the ictal or postictal phenomenon including subjective symptoms during video-EEG monitoring and the stereotypic history from medical records, we classified their semiology to localized, lateralized or not helpful.

If possible, functional neuroimaging studies were performed as described previously. ${ }^{10}$ FDG-PET during the interictal period (no seizures for more than $24 \mathrm{~h}$ ) was performed in 143 patients. FDG-PET images were assessed visually and using statistical parametric map- 
ping (SPM) analyses. Ictal and interictal SPECT was performed in 101 patients. Side-by-side visual analysis of interictal and ictal images and a subtraction method were performed. The results of functional neuroimaging studies were defined as localizing if the predominant hypometabolic zone (FDG-PET) or the predominant hyperperfusion area (ictal-interictal SPECT) was confined to the epileptogenic lobe.

After these noninvasive studies, extraoperative intracranial monitoring was performed in all patients to define the resection margin. We used combinations of subdural grids and strips. The placement of the grids and strips was determined by the results of noninvasive studies (video-scalp EEG monitoring, FDG-PET, and ictal-interictal SPECT). We categorized the distribution of seizure onset as focal (involving $<5$ adjacent electrodes), regional (involving $<5$ adjacent electrodes), or widespread (involving $>20$ adjacent electrodes). The ictal onset zone of extraoperative intracranial monitoring was defined as the area with the first sustained rhythmic change in EEG that was differentiated from the background and interictal waves. When necessary, preoperative and intraoperative functional mapping and intraoperative electrocorticography were performed.

\section{Surgery and pathology}

The ictal onset zone and the area with frequent interictal spikes accompanied by persistent pathological delta slowing of extraoperative intracranial monitoring were included in the resection area (epileptogenic lobe), if possible. The resection margin was tailored to the location of the eloquent cortex. All operations were performed by one neurosurgeon (C. K. Chung). Tissue sections from operation were immersed and fixed in $10 \%$ buffered formalin, embedded in paraffin, and stained with hematoxylin and eosin, cresyl violet, and Bielschowsky stain.

\section{Surgical outcomes}

Outcome status was assessed via outpatient clinical interviews. The outcome was stratified according to Engel's classification: class 1, seizure free; class 2, $>90 \%$ reduction; class 3,>50\% reduction; and class $4,<50 \%$ reduction or unchanged. The surgical outcomes and follow-up duration were evaluated according to the most recent medical record. The recent outcome of 89 patients in old group did not change significantly as compared with previously reported outcome (mean Engel's classification 2.17 vs. 2.22, $p=0.759$ ). Six patients in old group were improved and five patients were aggravated according to the most recent medical record as compared with previously reported outcome.

Table 1. Characteristics of the two groups including 142 patients with nonlesional neocortical epilepsy

\begin{tabular}{|c|c|c|c|}
\hline & Old group $(n=89)$ & New group $(n=53)$ & $p$-value \\
\hline Male sex (\%) & $55(61.8)$ & $24(45.3)$ & 0.080 \\
\hline Age at surgery, mean (SD), years & $26.6(7.91)$ & $28.1(8.39)$ & 0.265 \\
\hline Age at onset of nonfebrile seizure, mean (SD), years & $13.1(7.65)$ & $13.9(8.66)$ & 0.556 \\
\hline Duration of epilepsy, mean (SD), years & $13.5(6.50)$ & $14.4(8.62)$ & 0.483 \\
\hline Localized/lateralized semiology (\%) & $54(60.7)$ & $31(58.5)$ & 0.860 \\
\hline Resection of Temporal lobe (\%) & $31(34.8)$ & $33(62.3)$ & $0.002^{*}$ \\
\hline Complete resection, ictal onset zone (\%) & $72(80.9)$ & $48(90.6)$ & 0.154 \\
\hline Complete resection, interictal irritative zone (\%) & $60(67.4)$ & $40(75.5)$ & 0.346 \\
\hline Focal cortical dysplasia (\%) & $53 / 80(66.3)$ & $41 / 52(78.8)$ & 0.168 \\
\hline No. of presurgical evaluations, ${ }^{a}$ mean (SD) & $3.52(0.605)$ & $3.68(0.471)$ & 0.077 \\
\hline \multicolumn{4}{|l|}{ No. of patients with presurgical evaluation } \\
\hline FDG-PET & $79(88.8 \%)$ & $53(100 \%)$ & $0.014^{*}$ \\
\hline Ictal/interictal SPECT & $56(62.9 \%)$ & $36(67.9 \%)$ & 0.589 \\
\hline Mean concordance of presurgical evaluation, ${ }^{a}$ mean (SD) & $1.72(1.138)$ & $2.79(0.689)$ & $<0.001^{*}$ \\
\hline \multicolumn{4}{|l|}{ Concordance, two or more } \\
\hline Total & $48(53.9 \%)$ & $53(100 \%)$ & $<0.001^{*}$ \\
\hline Neotemporal & $24 / 31(77.4 \%)$ & $33 / 33(100 \%)$ & 0.004 \\
\hline Extratemporal & $24 / 58(41.4 \%)$ & $20 / 20(100 \%)$ & $<0.001^{*}$ \\
\hline
\end{tabular}

Ictal scalp EEG, interictal scalp EEG, FDG-PET and ictal-interictal SPECT.

" statistically significant.

SD, standard deviation; FDG-PET, fluorodeoxyglucose-positron emission tomography; SPECT, single-photon emission computed tomography; Neotemporal, neocortical temporal. 


\section{Statistical analyses}

Data storage and statistical analyses were performed using SPSS software (19.0; IBM SPSS Statistics for Windows, Armonk, NY, USA). Student's $t$ test was used for the analysis of continuous variables. The $\chi^{2}$ test or Fisher's exact test of independence was used for univariate analyses. A stepwise multiple linear regression analysis was performed for multivariate analyses. Statistical significance was set at $p<0.05$.

\section{Results}

\section{Patient characteristics}

There were no differences in sex, age at surgery, onset of nonfebrile seizure, and disease duration between the old and the new groups (Table 1). Among the 153 patients included in the study, the mean age at surgery was 27.2 years (range, $7-56$ years) and $55.6 \%$ were male. The number of patients with localized or lateralized seizure semiology was not different between the two groups. After application of the new strategy, resection of neocortical temporal lobe

Table 2. Surgical outcomes of patients before and after the implementation of the new selection strategy

\begin{tabular}{lcrc}
\hline & Old group $(\mathrm{n}=89)$ & New group $(\mathrm{n}=53)$ & $p$-value \\
\hline Good outcome (EI-III) & $73(82 \%)$ & $52(98.1 \%)$ & $0.003^{*}$ \\
Neotemporal & $27 / 31(87.1 \%)$ & $32 / 33(97.0 \%)$ & 0.190 \\
Extratemporal & $46 / 58(79.3 \%)$ & $20 / 20(100 \%)$ & $0.030^{*}$ \\
Seizure free (EI) & $42(47.2 \%)$ & $40(75.5 \%)$ & $0.001^{*}$ \\
Neotemporal & $18 / 31(58.1 \%)$ & $27 / 33(81.8 \%)$ & 0.055 \\
Extratemporal & $24 / 58(41.4 \%)$ & $13 / 20(65 \%)$ & 0.077 \\
\hline
\end{tabular}

*statistically significant.

Neotemporal, neocortical temporal.

Table 3. Predictors of surgical outcome in the 153 patients included in the study (univariate analysis)

\begin{tabular}{|c|c|c|c|}
\hline & Seizure free $(n=84)$ & Persistent seizure $(n=69)$ & $p$-value \\
\hline \multicolumn{4}{|l|}{ Clinical variables } \\
\hline Male sex (\%) & $45(53.6)$ & $40(58.0)$ & 0.586 \\
\hline Age at surgery, mean (SD), years & $27.61(7.967)$ & $26.65(8.049)$ & 0.464 \\
\hline Age at onset of nonfebrile seizure, mean (SD), years & $14.15(7.832)$ & $12.26(8.094)$ & 0.145 \\
\hline Duration of epilepsy, mean (SD), years & $13.55(7.884)$ & $14.39(6.946)$ & 0.488 \\
\hline Localized/lateralized semiology (\%) & $52(61.9)$ & $42(60.9)$ & 0.896 \\
\hline \multicolumn{4}{|l|}{ Presurgical noninvasive studies } \\
\hline Interictal scalp EEG, localizing (\%) & $40(47.6)$ & $20(29.0)$ & $0.019^{*}$ \\
\hline PET, localizing (\%) & $58 / 82(70.7)$ & 23/61 (37.7) & $0.000^{*}$ \\
\hline Ictal-interictal SPECT, localizing (\%) & $37 / 54(68.5)$ & $21 / 47(44.7)$ & $0.016^{*}$ \\
\hline Ictal scalp EEG, localizing (\%) & $70(83.3)$ & $40(58.0)$ & $0.001^{*}$ \\
\hline Concordance, two or more (\%) & $70(83.3)$ & $31(44.9)$ & $0.000^{*}$ \\
\hline \multicolumn{4}{|l|}{ Invasive studies } \\
\hline Focal onset pattern of invasive study (\%) & $40(47.6)$ & $28(40.6)$ & 0.412 \\
\hline Beta & $46(54.8)$ & $45(65.2)$ & \\
\hline Alpha & $13(15.5)$ & $7(10.1)$ & \\
\hline \multirow{4}{*}{$\begin{array}{l}\text { Theta } \\
\text { Delta } \\
\text { Periodic spike and wave }\end{array}$} & $8(9.5)$ & $5(7.2)$ & 0.510 \\
\hline & $6(7.1)$ & $7(10.1)$ & \\
\hline & $11(13.1)$ & $5(7.2)$ & \\
\hline & & & \\
\hline Neotemporal resection (\%) & $46(54.8)$ & $20(29)$ & $0.002^{*}$ \\
\hline Complete resection, ictal onset zone (\%) & $71(84.5)$ & $57(82.6)$ & 0.827 \\
\hline Complete resection, interictal irritative zone (\%) & $64(76.2)$ & $43(62.3)$ & 0.077 \\
\hline Focal cortical dysplasia (\%) & $56 / 80(70)$ & $48 / 63(76.2)$ & 0.453 \\
\hline
\end{tabular}

\footnotetext{
* statistically significant.
}

SD, standard deviation; EEG, electroencephalogram; PET, positron emission tomography; SPECT, single-photon emission computed tomography; Neotemporal, neocortical temporal. 
Table 4. Variables associated with seizure free outcome in multivariate analyses

\begin{tabular}{lccc}
\hline Variable & Odds ratio & $95 \%$ confidence interval & $p$-value \\
\hline Concordance, two or more & 5.612 & $1.612-19.538$ & 0.007 \\
PET, localizing & 3.715 & $1.256-10.994$ & 0.018 \\
\hline
\end{tabular}

Adjusted age at surgery, age at onset of nonfebrile seizure, follow-up period, localized/lateralized semiology, localizing interictal EEG, localizing ictal EEG, localizing PET, localizing ictal-interictal SPECT, resection of neocortical temporal lobe as independent variables.

$\mathrm{PET}$, positron emission tomography.

Table 5. Localizing value of individual presurgical modalities according to the resected lobe in seizure-free patients (84 patients)

\begin{tabular}{lcccc}
\hline & Interictal EEG & Ictal EEG & PET & Ictal-interictal SPECT \\
\hline Frontal (\%) & $12 / 25(48)$ & $22 / 25(88)$ & $11 / 24(45.8)$ & $10 / 17(58.8)$ \\
Neotemporal (\%) & $23 / 46(50)$ & $38 / 46(82.6)$ & $41 / 45(91.1)$ & $22 / 26(84.6)$ \\
Parietal (\%) & $0 / 4$ & $1 / 4(25)$ & $2 / 4(50)$ & $1 / 3(33.3)$ \\
Occipital (\%) & $5 / 9(55.6)$ & $9 / 9(100)$ & $4 / 9(44.4)$ & $4 / 8(50)$ \\
Total (\%) & $40 / 84(47.6)$ & $70 / 84(83.3)$ & $58 / 82(70.7)$ & $37 / 54(68.5)$ \\
pralue $^{a}$ & 0.307 & $0.024^{*}$ & $<0.000^{*}$ & 0.054 \\
\hline
\end{tabular}

${ }^{a}$ Fisher's exact test.

EEG, electroencephalogram; PET, positron emission tomography; SPECT, single-photon emission computed tomography.

was increased (34.8\% vs $62.3 \% ; p=0.002$ ). There were 53 cases of cortical dysplasia in the old group and 41 cases in the new group. The percentage of cortical dysplasia was not different between two groups.

\section{Presurgical evaluations and their concordance}

The number of presurgical evaluations was not different between patients of the new group and the old group (Table 1). However the number of patients with FDG-PET was significantly high in the new group ( $88.8 \%$ vs. $100 \% ; p=0.014$ ). The mean concordance of presurgical evaluations (range, $0-4$ ) and the number of patients with concordance between two or more evaluations were higher in the new group compared with the old group. The concordance among presurgical evaluations for extratemporal resection increased after implementation of new strategy.

\section{Surgical outcome according to the application of the new strategy}

Surgical outcome was improved after the application of the new strategy (Table 2). In the old group, 73 (82\%) patients had a good surgical outcome (EI-III) and 42 (47.2\%) patients were seizure free. Compared with the old group, a higher percentage of patients in the new group had a good surgical outcome (52 patients, $98.1 \% ; p=0.003$ ), including 40 patients who were seizure free $(75.5 \% ; p=0.001)$.

\section{Predictors of surgical outcome}

The univariate analysis revealed that sex, age at surgery, age at onset of nonfebrile seizure, duration of epilepsy, and localized/lateralized semiology were not significant prognostic factors of outcome (Table 3). In terms of presurgical evaluation, localizing interictal scalp $\operatorname{EEG}(p=0.019)$, FDG-PET $(p=0.000)$, ictal-interictal SPECT ( $p=$ $0.016)$, and ictal scalp EEG $(p=0.001)$ were associated with a seizure-free outcome. Concordance between two or more modalities was also related to seizure-free outcome $(p=0.000)$. The parameters of invasive studies, including onset pattern and onset frequency of ictal discharge, were not related to surgical outcome. Neocortical temporal location of surgical resection was a good prognostic factor $(p=0.002)$. Binominal multiple logistic regression identified only two or more concordant modalities $(p=0.007)$ and localizing FDG-PET $(p=0.018)$ as independent prognostic factors of seizure-free outcome (Table 4).

Localizing value of individual presurgical modalities according to the resected lobe was estimated (Table 5). The localizing value of FDG-PET was greatest in neocortical TLE. The predictive value of noninvasive presurgical modalities was estimated (Supplementary Table 1). FDG-PET showed higher positive predictive value (0.72) rather than other modalities.

\section{Discussion}

There is an inherent difficulty in identifying the epileptogenic zone in MRI-negative neocortical epilepsy, which leads to incomplete resection. Until recently, only a minority of these patients were seizure-free after resection. Seizure-free outcomes range from 31\% to 
70\% for MRI-negative TLE and from 17\% to 57\% for MRI-negative extratemporal lobe epilepsy. ${ }^{10-15}$ Two large series ${ }^{10,13}$ demonstrated that seizure-free outcomes are $47 \%$ and $55 \%$ for MRI-negative TLE and $41 \%$ and $43 \%$ for MRI-negative extratemporal lobe epilepsy, respectively. A recent meta-analysis demonstrated that the seizure-free rates of nonlesional TLE and extratemporal lobe epilepsy were $45 \%$ and $26 \%$, respectively, which were significantly lower compared with those observed in lesional epilepsies (72\% and 53\%, respectively). ${ }^{9}$ In this study, we demonstrated improved surgical outcome after application of the new selection strategy. The seizure-free outcome increased from $47.2 \%$ to $75.5 \%$. This is comparable with that of the lesional epilepsy group.

We found that the complete resection of intracranial ictal onset zone or significant interictal activities was not related with seizure-free outcome. There are some studies that demonstrated completeness of resection as a prognostic factor of epilepsy surgery. ${ }^{13,16,17}$ This inconsistent result may be partially due to the different definition of completeness of resection. They defined complete resection using not only findings of intracranial EEG but also results of $M R I^{16}$ or of other presurgical evaluations. ${ }^{13}$

Because a subset of remote propagated electrographic seizures represents independent initiation of secondary electrographic seizures indistinguishable from those at the local onset side, ${ }^{18}$ the focal intracranial ictal fast activity does not necessarily mean the true ictal onset zone. ${ }^{19}$ Even complete resection of the area with intracranial ictal onset zone can cause poor surgical outcome when the hypothesis is based on the wrong assumption which is derived from the less concordant presurgical evaluations.

In addition to the concordance, each presurgical evaluation (interictal scalp EEG, ictal scalp EEG, FDG-PET, and ictal-interictal SPECT) was associated with seizure-free outcome. However, a multivariate analysis showed that FDG-PET and concordance were significant prognostic factors.

Possible mechanisms of FDG-PET hypometabolism are as follows: atrophy or partial volume effects, neuronal loss, hypometabolic macro- or microscopic lesions, decreased synaptic activity, deafferentation because of reduced number of synapses, postictal metabolic depression, and inhibitory mechanism of seizures. ${ }^{20}$ Compared with the mechanism of ictal SPECT as a simple physiological change accompanied by ictal discharges, most of possible mechanisms of FDG-PET reflect the pathogenesis of epilepsy. In other words, FDG-PET imaging can be regarded not only as functional, but also as partial structural imaging. This can explain the independent prog- nostic value of FDG-PET found in our study. A recent study reported that FDG-PET improved surgical outcome in focal cortical dysplasia type IIB with negative MRI. ${ }^{21}$ Recent studies showed similar outcome of epileptic surgery between medial temporal sclerosis group and nonlesional temporal epilepsy group with localizing FDG-PET. $^{22,23}$ The predictive value of FDG-PET regarding seizure freedom reached $86 \%$.

FDG-PET and subtraction SPECT are valuable in the diagnosis of MRI-negative neocortical epilepsy. ${ }^{24,25}$ However, in patients with intracranial ictal onset zones located outside the temporal lobe, their value is limited. FDG-PET and subtraction SPECT have localizing value in no more than half of patients, and occasionally yield false localizations. The overall sensitivity of FDG-PET in FLE was reported to be $46 \%$ to $96 \%{ }^{20}$ There is a correlation between the presence of lesions on MRI and positive PET scan results. Localizing PET abnormalities can be found in only $29 \%$ to $45 \%$ of MRI-negative frontal lobe epilepsy patients. ${ }^{10,26-28}$ Compared with FLE, FDG-PET is useful in MRI-negative TLE patients. One study demonstrated that 26 out of 30 patients with TLE without hippocampal sclerosis had concordant FDG-PET lateralization. ${ }^{29}$ In our series, 41 out of 45 patients (91.1\%) with MRI-negative TLE showed correct localizing FDG-PET results (Table 5). The high diagnostic value of FDG-PET in nonlesional TLE may contribute to the high concordance of presurgical evaluations and allow the recruitment of these patients for epilepsy surgery. The higher proportion of patients with nonlesional TLE in the new group was at least partly explained by the easy recruitment of these patients based on the higher concordance. Techniques such as FDG-PET/MRI coregistration or SPM may yield better results compared with visual analysis. ${ }^{21,30}$

The localizing value of ictal scalp EEG has not been studied extensively in neocortical focal epilepsy. It frequently does not localize (and can localize falsely) lesions in cases in which the focus is very circumscribed is located in the depths of a sulcus. ${ }^{31-34}$ Many previous studies used small numbers of patients or were confined to specific epileptic syndromes. Two studies showed the clinical usefulness of ictal surface EEG for neocortical epilepsies. ${ }^{35,36}$ Ictal scalp EEGs yield correct localization in $50.2 \%$ of extratemporal epilepsy cases and $74.5 \%$ of neocortical TLE cases. ${ }^{36}$ We reported previously that $42 \%$ of ictal scalp EEGs are localizable in neocortical epilepsy cases and that $62 \%$ of patients have at least one localizable ictal scalp EEG. ${ }^{35}$ Short seizure duration, fast cortical spreading, and frequent muscle artifact in seizures with early motor phenomenon, as well as the fact that a large portion of the frontal lobe cortex is inaccessible to scalp 
electrodes, limit the value of scalp EEG in FLE. ${ }^{35}$

The diagnostic accuracy of ictal scalp EEG in our study was as high as $83.3 \%$. However, the role of ictal scalp EEG in MRI-negative epilepsy is difficult to interpret because there should be a selection bias resulting from the easier recruitment for surgery of patients with localized ictal EEG, especially in the absence of a structural lesion.

The correct rate of localization of interictal scalp EEG in our study was $47.6 \%$. The values were similar among FLE, neo-TLE, and PLE. However, in PLE, no correct localization was possible. In our previous study, interictal scalp EEG did not localize the epileptogenic lobe correctly in all 14 patients with PLE who became seizure-free after surgery. ${ }^{37}$ Interictal scalp EEGs in PLE were frequently normal or nonlateralized, or even falsely localized to the temporal or frontal lobe in PLE. Patients with focal interictal spikes in the neocortical epileptogenic areas are a minority. ${ }^{10,31,38}$ Interictal scalp EEG identifies epileptiform discharges in $60 \%$ to $80 \%$ of patients with $F L E$, but these abnormalities have a lower localizing value compared with what is observed in TLE. ${ }^{32,34,39-41}$ The localizing value of EEG findings is higher in lateral FLE than it is in medial or basal FLE. The presence of focal interictal spikes in MRI-negative epilepsy is associated with a good surgical outcome. ${ }^{10,13}$ Poor outcome is predicted by the presence of spikes distant from the resected lobe, multiple spikes, or generalized spike-and-wave patterns. ${ }^{12,42}$

In our series, the frequency of ictal discharge found on intracranial electrodes was not related to surgical outcome. The relationship between the frequency detected by intracranial electrodes and surgical outcome was controversial. A variety of electrographic intracranial seizure onset patterns have been described. ${ }^{18,35}$ However, the first electrographic change does not always indicate a true ictal onset zone, and some patterns represent a propagated phenomenon. ${ }^{18,35}$ The surgical outcome depends strongly on the identification and complete resection of a well-defined true epileptogenic zone. ${ }^{13,43}$ Some studies suggested that focal fast activity at onset on intracranial electrodes are associated with a favorable surgical outcome in nonlesional extratemporal lobe epilepsy patients. ${ }^{44-47}$ It has been postulated that electrodes showing a high frequency at seizure onset might be close to the true ictal onset zone. ${ }^{31,48}$ However, other studies reported contradictory results. ${ }^{16,18}$ Many propagation rhythms were also within the fast frequency. ${ }^{19}$ It was hypothesized that a subset of remote propagated electrographic seizures represented an independent initiation of secondary electrographic seizures that were indistinguishable from those at the local onset site. ${ }^{18}$ Furthermore, it was also important to resect areas with specific intracranial interictal patterns, as well as the ictal onset zone, to obtain a good surgical outcome. ${ }^{16}$ These findings might explain in part the controversial results regarding the relationship between the intracranial ictal onset frequencies and surgical outcome.

In the future, it will be necessary to evaluate the possible roles of the newly introduced techniques, such as high frequency oscillations of EEG, diffusion tensor imaging, magnetoencephalogram (MEG), or PET scans with new ligands. They may enhance the diagnostic concordance and expand the number of surgical candidates.

This was the largest study based on epilepsy surgery of MRI-negative neocortical epilepsy patients performed to date. However, there are some limitations of this study. We cannot exclude the effect of learning curve of surgeon and the multidisciplinary epilepsy surgery team in spite we exclude the patients of very early period. The improvement of equipment (i.e. the more use of 3T MRI in new group while most patients in old group used 1.5T MRI) or processing software can affect the results of presurgical evaluation and surgical outcome. Although there were no significant change of re-evaluated outcome of old group, the relatively short follow-up duration of new group can be related to good outcome. We did not evaluate newly introduced methods such as MEG that could possibly affect the decision of surgery and surgical outcome. Finally, the retrospective design of this study limits the power of findings.

\section{Conclusions}

In conclusion, the new selection strategy to select the MRI-negative neocortical epilepsy patients with two or more concordant results of presurgical noninvasive studies led to a significant increase in surgical outcome (seizure-free outcome, $47.2 \%$ vs. $75.5 \% ; p=0.001$ ). Concordance between two or more presurgical evaluations and localizing PET were significantly related to a seizure-free outcome in a multivariate analysis. MRI-negative neocortical epilepsy patients with two or more concordance between presurgical noninvasive studies seem to be good candidates for nonlesional neocortical epilepsy surgery.

\section{Acknowledgement}

This study was supported by the Ministry of Health and Welfare (A070001-1232-1280100). 


\section{References}

1. Lee SK, Choe G, Hong KS, et al. Neuroimaging findings of cortical dyslamination with cytomegaly. Epilepsia 2001;42:850-6.

2. Bien $C G$, Szinay $M$, Wagner J, Clusmann $H$, Becker $A J$, Urbach $H$. Characteristics and surgical outcomes of patients with refractory magnetic resonance imaging-negative epilepsies. Arch Neurol 2009;66:1491-9.

3. Jeha $L E$, Najm I, Bingaman W, Dinner D, Widdess-Walsh P, Luders $H$. Surgical outcome and prognostic factors of frontal lobe epilepsy surgery. Brain 2007;130:574-84.

4. Spencer SS. Long-term outcome after epilepsy surgery. Epilepsia 1996;37:807-13.

5. West S, Nolan SJ, Cotton J, et al. Surgery for epilepsy. Cochrane Database Syst Rev 2015;(7):CD010541.

6. Van Gompel JJ, Ottman R, Worrell GA, et al. Use of anterior temporal lobectomy for epilepsy in a community-based population. Arch Neurol 2012;69:1476-81.

7. Kumlien E, Mattsson P. Attitudes towards epilepsy surgery: A nationwide survey among Swedish neurologists. Seizure 2010;19:253-5.

8. Brodie M, Barry SJ, Bamagous GA, Norrie JD, Kwan P. Patterns of treatment response in newly diagnosed epilepsy. Neurology 2012;78:1548-54.

9. Tellez-Zenteno JF, Hernandez Ronquillo L, Moien-Afshari F, Wiebe S. Surgical outcomes in lesional and non-lesional epilepsy: a systematic review and meta-analysis. Epilepsy Res 2010;89:310-8.

10. Lee SK, Lee SY, Kim KK, Hong KS, Lee DS, Chung CK. Surgical outcome and prognostic factors of cryptogenic neocortical epilepsy. Ann Neurol 2005;58:525-32.

11. Siegel AM, Jobst BC, Thadani VM, et al. Medically intractable, localization-related epilepsy with normal MRI: presurgical evaluation and surgical outcome in 43 patients. Epilepsia 2001;42:883-8.

12. Blume WT, Ganapathy GR, Munoz D, Lee DH. Indices of resective surgery effectiveness for intractable nonlesional focal epilepsy. Epilepsia 2004;45:46-53.

13. Jayakar $P$, Dunoyer $C$, Dean $P$, et al. Epilepsy surgery in patients with normal or nonfocal MRI scans: integrative strategies offer long-term seizure relief. Epilepsia 2008;49:758-64.

14. Noe K, Sulc V, Wong-Kisiel L, et al. Long-term outcomes after nonlesional extratemporal lobe epilepsy surgery. JAMA Neurol 2013;70:1003-8.

15. See SJ, Jehi LE, Vadera S, Bulacio J, Najm I, Bingaman W. Surgical outcomes in patients with extratemporal epilepsy and subtle or normal magnetic resonance imaging findings. Neurosurgery 2013;73:68-76; discussion 76-7.

16. Kim DW, Kim HK, Lee SK, Chu K, Chung CK. Extent of neocortical resection and surgical outcome of epilepsy: intracranial EEG analysis. Epilepsia 2010;51:1010-7.

17. Wang $X$, Zhang $C$, Wang $Y$, et al. Prognostic factors for seizure outcome in patients with MRI-negative temporal lobe epilepsy: A meta-analysis and systematic review. Seizure 2016;38:54-62.

18. Schiller $Y$, Cascino GD, Busacker NE, Sharbrough FW. Characterization and comparison of local onset and remote propagated electrographic seizures recorded with intracranial electrodes. Epilepsia 1998;39:380-8.

19. Lee SK, Kim KK, Nam H, Oh JB, Yun CH, Chung CK. Adding or repositioning intracranial electrodes during presurgical assessment of neocortical epilepsy: electrographic seizure pattern and surgical outcome. J Neurosurg 2004;100:463-71.

20. Mauguiere F, Ryvlin P. The role of PET in presurgical assessment of partial epilepsies. Epileptic Disord 2004;6:193-215.

21. Chassoux F, Rodrigo $S$, Semah $F$, et al. FDG-PET improves surgical outcome in negative MRI Taylor-type focal cortical dysplasias. Neurology 2010;75:2168-75.

22. Yang PF, Pei JS, Zhang HJ, et al. Long-term epilepsy surgery outcomes in patients with PET-positive, MRI-negative temporal lobe epilepsy. Epilepsy Behav 2014;41:91-7.

23. LoPinto-Khoury C, Sperling MR, Skidmore C, et al. Surgical outcome in PET-positive, MRI-negative patients with temporal lobe epilepsy. Epilepsia 2012;53:342-8.

24. Henry TR, Babb TL, Engel J Jr, Mazziotta JC, Phelps ME, Crandall PH. Hippocampal neuronal loss and regional hypometabolism in temporal lobe epilepsy. Ann Neurol 1994;36:925-7.

25. Duncan R, Biraben A, Patterson J, et al. Ictal single photon emission computed tomography in occipital lobe seizures. Epilepsia 1997;38:839-43.

26. Ryvlin P, Bouvard S, Le Bars D, et al. Clinical utility of flumazenil-PET versus [18F]fluorodeoxyglucose-PET and MRI in refractory partial epilepsy. A prospective study in 100 patients. Brain 1998;121(Pt 11):2067-81.

27. Kim YK, Lee DS, Lee SK, Chung CK, Chung JK, Lee MC. (18)F-FDG PET in localization of frontal lobe epilepsy: comparison of visual and SPM analysis. J Nucl Med 2002;43:1167-74.

28. Swartz BE, Brown C, Mandelkern MA, et al. The use of 2-deoxy-2-[18F]fluoro-D-glucose (FDG-PET) positron emission tomography in the routine diagnosis of epilepsy. Mol Imaging Biol 2002;4:245-52.

29. Carne RP, O'Brien TJ, Kilpatrick CJ, et al. MRI-negative PET-positive temporal lobe epilepsy: a distinct surgically remediable syndrome. Brain 2004;127:2276-85.

30. Plotkin M AH, Merschhemke M, Lüdemann $L$, et al. Use of statistical parametric mapping of (18) F-FDG-PET in frontal lobe epilepsy. Nuklearmedizin 2003;42:190-6.

31. Williamson PD, Thadani VM, Darcey TM, Spencer DD, Spencer SS, Mattson RH. Occipital lobe epilepsy: clinical characteristics, seizure spread patterns, and results of surgery. Ann Neurol 1992;31:3-13.

32. Salanova V, Morris HH 3rd, Van Ness PC, Luders H, Dinner D, Wyllie E. Comparison of scalp electroencephalogram with subdural electrocorticogram recordings and functional mapping in frontal lobe epilepsy. Arch Neurol 1993;50:294-9.

33. Palmini A, Andermann F, Dubeau F, et al. Occipitotemporal epilepsies: evaluation of selected patients requiring depth electrodes studies and rationale for surgical approaches. Epilepsia 1993;34:84-96.

34. Laskowitz DT, Sperling MR, French JA, O'Connor MJ. The syndrome of 
frontal lobe epilepsy: characteristics and surgical management. Neurology 1995;45:780-7.

35. Lee SK, Kim JY, Hong KS, Nam HW, Park SH, Chung CK. The clinical usefulness of ictal surface EEG in neocortical epilepsy. Epilepsia 2000;41:1450-5.

36. Foldvary N, Klem G, Hammel J, Bingaman W, Najm I, Luders H. The localizing value of ictal EEG in focal epilepsy. Neurology 2001;57:2022-8.

37. Kim DW, Lee SK, Yun CH, et al. Parietal lobe epilepsy: the semiology, yield of diagnostic workup, and surgical outcome. Epilepsia 2004;45:641-9.

38. Pathak P BW. Asymmetrical epileptogenicity of brain lesions. 1997; Proceeding of annual meeting of American Epilepsy Society.

39. Mosewich RK, So EL, O'Brien TJ, et al. Factors predictive of the outcome of frontal lobe epilepsy surgery. Epilepsia 2000;41:843-9.

40. Wetjen NM, Cohen-Gadol AA, Maher CO, Marsh WR, Meyer FB, Cascino GD. Frontal lobe epilepsy: diagnosis and surgical treatment. Neurosurg Rev 2002;25:119-38; discussion 139-40.

41. Kellinghaus C, Luders HO. Frontal lobe epilepsy. Epileptic Disord 2004:6:223-39.

42. Sunwoo JS, Byun JI, Moon J, et al. Unfavorable surgical outcomes in partial epilepsy with secondary bilateral synchrony: Intracranial electroencephalography study. Epilepsy Res 2016;122:102-9.

43. Kim DW, Lee SK, Chu K, et al. Predictors of surgical outcome and pathologic considerations in focal cortical dysplasia. Neurology 2009;72:211-6.

44. Zakaria T, Noe K, So E, et al. Scalp and intracranial EEG in medically intractable extratemporal epilepsy with normal MRI. ISRN Neurol 2012;2012:942849.

45. Park SA, Lim SR, Kim GS, et al. Ictal electrocorticographic findings related with surgical outcomes in nonlesional neocortical epilepsy. Epilepsy Res 2002;48:199-206.

46. Wetjen NM, Marsh WR, Meyer FB, et al. Intracranial electroencephalography seizure onset patterns and surgical outcomes in nonlesional extratemporal epilepsy. J Neurosurg 2009;110:1147-52.

47. Jimenez-Jimenez $D$, Nekkare $R$, Flores $L$, et al. Prognostic value of intracranial seizure onset patterns for surgical outcome of the treatment of epilepsy. Clin Neurophysiol 2015;126:257-67.

48. Fisher RS, Webber WR, Lesser RP, Arroyo S, Uematsu S. High-frequency EEG activity at the start of seizures. I Clin Neurophysiol 1992;9:441-8. 